\title{
Quintic B-Spline Collocation Method for Tenth Order Boundary Value Problems
}

\author{
K.N.S.Kasi Viswanadham \\ Department of Mathematics \\ National Institute of Technology \\ Warangal - 506004 (INDIA)
}

\author{
Y.Showri Raju \\ Department of Mathematics \\ National Institute of Technology \\ Warangal - 506004 (INDIA)
}

\begin{abstract}
A finite element method involving collocation method with quintic B-splines as basis functions has been developed to solve tenth order boundary value problems. The fifth order, sixth order, seventh order, eighth order, ninth order and tenth order derivatives for the dependent variable are approximated by the central differences of fourth order derivatives. The basis functions are redefined into a new set of basis functions which in number match with the number of selected collocated points in the space variable domain. The proposed method is tested on several linear and non-linear boundary value problems. The solution of a non-linear boundary value problem has been obtained as the limit of a sequence of solutions of linear boundary value problems generated by quasilinearization technique. Numerical results obtained by the present method are in good agreement with the exact solutions available in the literature.
\end{abstract}

\section{Keywords}

Collocation Method; Quintic B-spline; Basis Function; Tenth Order Boundary Value Problem; Absolute Error.

\section{INTRODUCTION}

The higher order boundary value problems are known to arise in hydrodynamic, hydro magnetic stability and applied sciences. It is well known that when a layer of fluid is heated from below and is subject to the action of rotation, instability may set in as ordinary convection which may be modelled by a tenth-order boundary value problem. In addition, ultrasonically assisted development of resists feature on semiconductor substrate is a popular development technique, but is difficult to understand. During this development process the developer i.e. 1:3 Methyl isobutyl Ketene and Isopropyl alcohol is heated because of ultrasonic agitation. During heating an infinite horizontal layer of fluid and then subjecting to the action of rotation, instability sets in. When this instability sets as an ordinary convection and a uniform magnetic field is also applied across the fluid in the same direction as gravity, then the problem is modelled by a tenthorder boundary value problem [1].

In this paper, we developed a collocation method with quintic B-splines as basis functions for getting the numerical solution of a general linear tenth order boundary value problem

\begin{abstract}
$a_{0}(x) y^{(10)}(x)+a_{1}(x) y^{(9)}(x)+a_{2}(x) y^{(8)}(x)+a_{3}(x) y^{(7)}(x)+$ $a_{4}(x) y^{(6)}(x)+a_{5}(x) y^{(5)}(x)+a_{6}(x) y^{(4)}(x)+a_{7}(x) y^{\prime \prime \prime}(x)+$ $a_{8}(x) y^{\prime \prime}(x)+a_{9}(x) y^{\prime}(x)+a_{10}(x) y(x)=b(x), \quad c<x<d$
\end{abstract}

subject to the boundary conditions

$$
\begin{array}{ll}
\mathrm{y}(\mathrm{c})=\mathrm{A}_{0}, & \mathrm{y}(\mathrm{d})=\mathrm{B}_{0}, \\
\mathrm{y}^{\prime}(\mathrm{c})=\mathrm{A}_{1}, & \mathrm{y}^{\prime}(\mathrm{d})=\mathrm{B}_{1}, \\
\mathrm{y}^{\prime \prime}(\mathrm{c})=\mathrm{A}_{2}, & \mathrm{y}^{\prime \prime}(\mathrm{d})=\mathrm{B}_{2}, \\
\mathrm{y}^{\prime \prime \prime}(\mathrm{c})=\mathrm{A}_{3}, & \mathrm{y}^{\prime \prime \prime}(\mathrm{d})=\mathrm{B}_{3}, \\
\mathrm{y}^{(4)}(\mathrm{c})=\mathrm{A}_{4}, & \mathrm{y}^{(4)}(\mathrm{d})=\mathrm{B}_{4}
\end{array}
$$

where $A_{0}, B_{0}, A_{1}, B_{1}, A_{2}, B_{2}, A_{3}, B_{3}, A_{4}, B_{4}$ are finite real constants and $\mathrm{a}_{0}(\mathrm{x}), \mathrm{a}_{1}(\mathrm{x}), \mathrm{a}_{2}(\mathrm{x}), \mathrm{a}_{3}(\mathrm{x}), \mathrm{a}_{4}(\mathrm{x}), \mathrm{a}_{5}(\mathrm{x}), \mathrm{a}_{6}(\mathrm{x}), \mathrm{a}_{7}(\mathrm{x})$, $a_{8}(x), a_{9}(x), a_{10}(x)$ and $b(x)$ are all continuous functions defined on the interval $[\mathrm{c}, \mathrm{d}]$.

The existence and uniqueness of the solution for these types of problems have been discussed in Agarwal [2]. The boundary value problems of higher order have been investigated because of both their mathematical importance and their potential for applications in hydro dynamic and hydro magnetic stability. Over the years, there are several authors who worked on these types of boundary value problems by using different methods. For example, Twizell et. al.[3] developed numerical methods for eighth, tenth and twelfth-order eigen value problems arising in thermal instability. Siddiqi and Twizell [4] presented the solution of tenth-order boundary value problem using tenth degree spline. Siddiqi and Akram [5] developed the solution of tenth-order boundary value problems using non-polynomial spline technique. Siddiqi and Akram [6] presented the solution of tenth-order boundary value problem by using eleventh degree spline. Erturk and Momani [7] applied differential transform method to construct the numerical solutions for linear and nonlinear tenth-order boundary value problems with two-point boundary conditions. Variational iteration technique was used by Siddiqi, Ghazala Ikram and Sabahat Zaheer [8] for the solution of tenth order boundary value problems. Homotopy Perturbation Method was used by Barari et. al.[9] for solving tenth order boundary value problems. Further, Scott and Watts [10] developed a numerical method for the solution of linear boundary value problems using a combination of superposition and orthonormalization. Scott and Watts [11] described several computer codes that were developed using the superposition and orthonormalization technique and invariant imbedding. Watson and Scott [12] proved that Chow-Yorke algorithm was globally convergent for a class of spline collocation approximations to non-linear two point 
boundary value problems. Solution of linear and nonlinear boundary value problems of tenth and twelfth-order was implemented by Wazwaz [13] using adomian decomposition method. Numerical methods for special nonlinear boundary value problems of order $2 \mathrm{~m}$ are developed by Djidjeli et. al.[14].

The above studies are concerned to solve tenth order boundary value problems by using tenth or eleventh order Bsplines. In this paper, quintic B-splines as basis functions have been used to solve the boundary value problems of the type (1)-(2).

In section 2 of this paper, the justification for using the collocation method has been mentioned. In section 3 , the definition of quintic B-splines has been described. In section 4 , description of the collocation method with quintic B-splines as basis functions has been presented and in section 5, solution procedure to find the nodal parameters is presented. In section 6, numerical examples of both linear and non-linear boundary value problems are presented. The solution of a nonlinear boundary value problem has been obtained as the limit of a sequence of solutions of linear boundary value problems generated by quasilinearization technique [15]. Finally, the last section is dealt with conclusions of the paper.

\section{JUSTIFICATION FOR USING COLLOCATION METHOD}

In finite element method (FEM) the approximate solution can be written as a linear combination of basis functions which constitute a basis for the approximation space under consideration. FEM involves variational methods such as Ritz's approach, Galerkin's approach, least squares method and collocation method etc. The collocation method seeks an approximate solution by requiring the residual of the differential equation to be identically zero at $\mathrm{N}$ selected points in the given space variable domain where $\mathrm{N}$ is the number of basis functions in the basis [16]. That means, to get an accurate solution by the collocation method, one needs a set of basis functions which in number match with the number of collocation points selected in the given space variable domain. Further, the collocation method is the easiest to implement among the variational methods of FEM. When a differential equation is approximated by $\mathrm{m}^{\text {th }}$ order B-splines, it yields $(\mathrm{m}+1)^{\text {th }}$ order accurate results [17]. Hence this motivated us to solve a tenth order boundary value problem of type (1)-(2) by collocation method with quintic B-splines as basis functions.

\section{DEFINITION OF QUINTIC B-SPLINES}

The cubic B-splines are defined in [18, 19]. In a similar analogue, the existence of the quintic spline interpolate $\mathrm{s}(\mathrm{x})$ to a function in a closed interval [c, d] for spaced knots (need not be evenly spaced) $\mathrm{c}=\mathrm{x}_{0}<\mathrm{x}_{1}<\mathrm{x}_{2}<\ldots<\mathrm{x}_{\mathrm{n}-1}<\mathrm{x}_{\mathrm{n}}=\mathrm{d}$ is established by constructing it. The construction of $s(x)$ is done with the help of the quintic B-splines. Introduce ten additional knots $\mathrm{x}_{-5}, \mathrm{x}_{-4}, \mathrm{x}_{-3}, \mathrm{x}_{-2}, \mathrm{x}_{-1}, \mathrm{x}_{\mathrm{n}+1}, \mathrm{x}_{\mathrm{n}+2}, \mathrm{x}_{\mathrm{n}+3}, \mathrm{x}_{\mathrm{n}+4}$ and $\mathrm{x}_{\mathrm{n}+5}$ such that

$$
\begin{aligned}
& \mathrm{x}_{-5}<\mathrm{x}_{-4}<\mathrm{x}_{-3}<\mathrm{x}_{-2}<\mathrm{x}_{-1}<\mathrm{x}_{0} \\
& \text { and } \mathrm{x}_{\mathrm{n}}<\mathrm{x}_{\mathrm{n}+1}<\mathrm{x}_{\mathrm{n}+2}<\mathrm{x}_{\mathrm{n}+3}<\mathrm{x}_{\mathrm{n}+4}<\mathrm{x}_{\mathrm{n}+5} .
\end{aligned}
$$

Now the quintic B-splines $\mathrm{B}_{\mathrm{i}}(\mathrm{x})$ 's are defined by

$$
\mathrm{B}_{\mathrm{i}}(x)= \begin{cases}\sum_{r=i-3}^{i+3} \frac{\left(x_{r}-x\right)^{5}+}{\pi^{\prime}\left(x_{r}\right)} & \text { if } \mathrm{x} \in\left[\mathrm{x}_{\mathrm{i}-3}, \mathrm{x}_{\mathrm{i}+3}\right] \\ 0 & \text { otherwise }\end{cases}
$$

where

$$
\begin{aligned}
& \left(\mathrm{x}_{\mathrm{r}}-\mathrm{x}\right)^{5}+= \begin{cases}\left(\mathrm{x}_{\mathrm{r}}-\mathrm{x}\right)^{5} & \text { if } \mathrm{x}_{\mathrm{r}} \geq \mathrm{x} \\
0 & \text { if } \mathrm{x}_{\mathrm{r}} \leq \mathrm{x}\end{cases} \\
& \text { and } \pi(\mathrm{x})=\prod_{r=i-3}^{i+3}\left(\mathrm{x}-\mathrm{x}_{\mathrm{r}}\right)^{\circ}
\end{aligned}
$$

It can be shown that the set $\left\{\mathrm{B}_{-2}(\mathrm{x}), \mathrm{B}_{-1}(\mathrm{x}), \mathrm{B}_{0}(\mathrm{x}), \ldots, \mathrm{B}_{\mathrm{n}}(\mathrm{x})\right.$, $\left.\mathrm{B}_{\mathrm{n}+1}(\mathrm{x}), \mathrm{B}_{\mathrm{n}+2}(\mathrm{x})\right\}$ forms a basis for the space $S_{5}(\pi)$ of fifth degree polynomial splines. The quintic B-splines are the unique non-zero splines of smallest compact support with knots at

$\mathrm{x}_{-5}<\mathrm{x}_{-4}<\mathrm{x}_{-3}<\mathrm{x}_{-2}<\mathrm{x}_{-1}<\mathrm{x}_{0}<\ldots<\mathrm{x}_{\mathrm{n}}<\mathrm{x}_{\mathrm{n}+1}<\mathrm{x}_{\mathrm{n}+2}<\mathrm{x}_{\mathrm{n}+3}<$ $\mathrm{x}_{\mathrm{n}+4}<\mathrm{x}_{\mathrm{n}+5}$.

\section{DESCRIPTION OF THE METHOD}

To solve the boundary value problem (1)-(2) by the collocation method with quintic B- splines as basis functions, we define the approximation for $y(x)$ as

$\mathrm{y}(\mathrm{x})=\sum_{j=-2}^{n+2} \alpha_{j} \mathrm{~B}_{\mathrm{j}}(\mathrm{x})$

where $\alpha_{j}$ s are nodal parameters to be determined. In the present method, the internal mesh points $\mathrm{x}_{3}, \mathrm{x}_{4}, \ldots, \mathrm{x}_{\mathrm{n}-3}$ are selected as the collocation points. In collocation method, the number of basis functions in the approximation should match with the number of collocation points[16]. Here the number of basis functions in the approximation (3) is $n+5$, where as the number of selected collocation points is $n-5$. So, there is a need to redefine the basis functions into a new set of basis functions which in number match with the number of selected collocation points. The procedure for redefining the basis functions is as follows:

Using the quintic B-splines described in section 3 and the Dirichlet boundary conditions of (2), we get the approximate solution at the boundary points as

$$
\begin{aligned}
& y(c)=\mathrm{y}\left(\mathrm{x}_{0}\right)=\sum_{j=-2}^{2} \alpha_{j} \mathrm{~B}_{\mathrm{j}}\left(\mathrm{x}_{0}\right)=A_{0} \\
& y(d)=\mathrm{y}\left(\mathrm{x}_{\mathrm{n}}\right)=\sum_{j=n-2}^{n+2} \alpha_{j} \mathrm{~B}_{\mathrm{j}}\left(\mathrm{x}_{\mathrm{n}}\right)=B_{0} .
\end{aligned}
$$

Eliminating $\alpha_{-2}$ and $\alpha_{n+2}$ from the equations (3), (4) and (5), we get the approximation for $\mathrm{y}(\mathrm{x})$ as

$$
\mathrm{y}(\mathrm{x})=\mathrm{w}_{1}(\mathrm{x})+\sum_{j=-1}^{n+1} \alpha_{j} \mathrm{P}_{\mathrm{j}}(\mathrm{x})
$$

where

$$
\mathrm{w}_{1}(\mathrm{x})=\frac{\mathrm{A}_{0}}{\mathrm{~B}_{-2}\left(\mathrm{x}_{0}\right)} \mathrm{B}_{-2}(\mathrm{x})+\frac{\mathrm{B}_{0}}{\mathrm{~B}_{\mathrm{n}+2}\left(\mathrm{x}_{\mathrm{n}}\right)} \mathrm{B}_{\mathrm{n}+2}(\mathrm{x})
$$

and 
$P_{j}(x)=\left\{\begin{array}{lrl}B_{j}(x)-\frac{B_{j}\left(x_{0}\right)}{B_{-2}\left(x_{0}\right)} B_{-2}(x), & \text { for } & j=-1,0,1,2 \\ B_{j}(x), & \text { for } & j=3,4, \ldots, n-3 \\ B_{j}(x)-\frac{B_{j}\left(x_{n}\right)}{B_{n+2}\left(x_{n}\right)} B_{n+2}(x), & \text { for } & j=n-2, n-1, n, n+1 .\end{array}\right.$.

Using the Neumann boundary conditions of (2) to the approximation $\mathrm{y}(\mathrm{x})$ in $(6)$, we get

$\mathrm{y}^{\prime}(\mathrm{c})=\mathrm{y}^{\prime}\left(\mathrm{x}_{0}\right)=\mathrm{w}_{1}{ }^{\prime}\left(\mathrm{x}_{0}\right)+\alpha_{-1} \mathrm{P}_{-1}{ }^{\prime}\left(\mathrm{x}_{0}\right)+\alpha_{0} \mathrm{P}_{0}{ }^{\prime}\left(\mathrm{x}_{0}\right)+\alpha_{1} \mathrm{P}_{1}{ }^{\prime}\left(\mathrm{x}_{0}\right)+$ $\alpha_{2} \mathrm{P}_{2}{ }^{\prime}\left(\mathrm{x}_{0}\right)=\mathrm{A}_{1}$

$\mathrm{y}^{\prime}(\mathrm{d})=\mathrm{y}^{\prime}\left(\mathrm{x}_{\mathrm{n}}\right)=\mathrm{w}_{1}{ }^{\prime}\left(\mathrm{x}_{\mathrm{n}}\right)+\alpha_{\mathrm{n}-2} \mathrm{P}_{\mathrm{n}-2}{ }^{\prime}\left(\mathrm{x}_{\mathrm{n}}\right)+\alpha_{\mathrm{n}-1} \mathrm{P}_{\mathrm{n}-1}{ }^{\prime}\left(\mathrm{x}_{\mathrm{n}}\right)+\alpha_{\mathrm{n}} \mathrm{P}_{\mathrm{n}}{ }^{\prime}\left(\mathrm{x}_{\mathrm{n}}\right)+$ $\alpha_{n+1} P_{n+1}^{\prime}\left(x_{n}\right)=B_{1}$.

Now, eliminating $\alpha_{-1}$ and $\alpha_{n+1}$ from the equations (6), (7) and (8), we get the approximation for $\mathrm{y}(\mathrm{x})$ as

$\mathrm{y}(\mathrm{x})=\mathrm{w}_{2}(\mathrm{x})+\sum_{j=0}^{n} \alpha_{j} \mathrm{Q}_{\mathrm{j}}(\mathrm{x})$

where

$\mathrm{w}_{2}(\mathrm{x})=\mathrm{w}_{1}(\mathrm{x})+\frac{\mathrm{A}_{1}-\mathrm{w}_{1}^{\prime}\left(\mathrm{x}_{0}\right)}{\mathrm{P}_{-1}^{\prime}\left(\mathrm{x}_{0}\right)} \mathrm{P}_{-1}(\mathrm{x})+\frac{\mathrm{B}_{1}-\mathrm{w}_{1}^{\prime}\left(\mathrm{x}_{\mathrm{n}}\right)}{\mathrm{P}_{\mathrm{n}+1}^{\prime}\left(\mathrm{x}_{\mathrm{n}}\right)} \mathrm{P}_{\mathrm{n}+1}(\mathrm{x})$

and

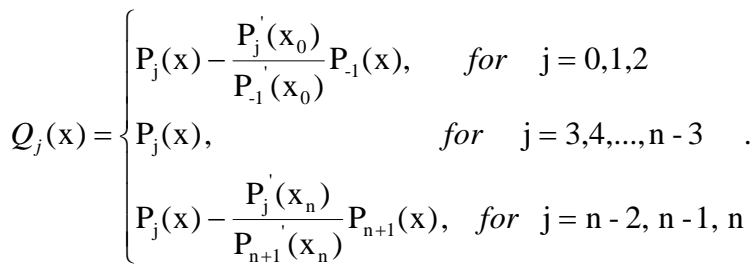

Using the boundary conditions $\mathrm{y}^{\prime \prime}(\mathrm{c})=\mathrm{A}_{2}$ and $\mathrm{y}^{\prime \prime}(\mathrm{d})=\mathrm{B}_{2}$ of (2) to the approximate solution $y(x)$ in (9), we get

$$
\begin{aligned}
\mathrm{y}^{\prime \prime}(\mathrm{c}) & =\mathrm{y}^{\prime \prime}\left(\mathrm{x}_{0}\right)=\mathrm{w}_{2}{ }^{\prime \prime}\left(\mathrm{x}_{0}\right)+\alpha_{0} \mathrm{Q}_{0}{ }^{\prime \prime}\left(\mathrm{x}_{0}\right)+\alpha_{1} \mathrm{Q}_{1}{ }^{\prime \prime}\left(\mathrm{x}_{0}\right)+\alpha_{2} \mathrm{Q}_{2}{ }^{\prime \prime}\left(\mathrm{x}_{0}\right) \\
& =\mathrm{A}_{2} \\
\mathrm{y}^{\prime \prime}(\mathrm{d}) & =\mathrm{y}^{\prime \prime}\left(\mathrm{x}_{\mathrm{n}}\right)=\mathrm{w}_{2}{ }^{\prime \prime}\left(\mathrm{x}_{\mathrm{n}}\right)+\alpha_{\mathrm{n}-2} \mathrm{Q}_{\mathrm{n}-2}{ }^{\prime \prime}\left(\mathrm{x}_{\mathrm{n}}\right)+\alpha_{\mathrm{n}-1} \mathrm{Q}_{\mathrm{n}-1}{ }^{\prime \prime}\left(\mathrm{x}_{\mathrm{n}}\right) \\
& +\alpha_{\mathrm{n}} \mathrm{Q}_{\mathrm{n}}{ }^{\prime \prime}\left(\mathrm{x}_{\mathrm{n}}\right)=\mathrm{B}_{2} .
\end{aligned}
$$

Now, eliminating $\alpha_{0}$ and $\alpha_{n}$ from the equations (9), (10) and (11), we get the approximation for $\mathrm{y}(\mathrm{x})$ as

$$
\mathrm{y}(\mathrm{x})=\mathrm{w}_{3}(\mathrm{x})+\sum_{j=1}^{n-1} \alpha_{j} \mathrm{R}_{\mathrm{j}}(\mathrm{x})
$$

where

$$
\begin{aligned}
& \mathrm{w}_{3}(\mathrm{x})=\mathrm{w}_{2}(\mathrm{x})+\frac{\mathrm{A}_{2}-\mathrm{w}_{2}{ }^{\prime \prime}\left(\mathrm{x}_{0}\right)}{\mathrm{Q}_{0}{ }^{\prime}\left(\mathrm{x}_{0}\right)} \mathrm{Q}_{0}(\mathrm{x}) \\
& +\frac{\mathrm{B}_{2}-\mathrm{w}_{2}{ }^{\prime \prime}\left(\mathrm{x}_{\mathrm{n}}\right)}{\mathrm{Q}_{\mathrm{n}}{ }^{\prime}\left(\mathrm{x}_{\mathrm{n}}\right)} \mathrm{Q}_{\mathrm{n}}(\mathrm{x})
\end{aligned}
$$

and

$$
R_{j}(x)=\left\{\begin{array}{l}
\mathrm{Q}_{\mathrm{j}}(\mathrm{x})-\frac{\mathrm{Q}_{\mathrm{j}}{ }^{\prime \prime}\left(\mathrm{x}_{0}\right)}{\mathrm{Q}_{0}{ }^{\prime}\left(\mathrm{x}_{0}\right)} \mathrm{Q}_{0}(\mathrm{x}), \text { for } \mathrm{j}=1,2 \\
\mathrm{Q}_{\mathrm{j}}(\mathrm{x}), \quad \text { for } \mathrm{j}=3,4, \ldots, \mathrm{n}-3 \\
\mathrm{Q}_{\mathrm{j}}(\mathrm{x})-\frac{\mathrm{Q}_{\mathrm{j}}{ }^{\prime \prime}\left(\mathrm{x}_{\mathrm{n}}\right)}{\mathrm{Q}_{\mathrm{n}}{ }^{\prime}\left(\mathrm{x}_{\mathrm{n}}\right)} \mathrm{Q}_{\mathrm{n}}(\mathrm{x}), \text { for } \mathrm{j}=\mathrm{n}-2, \mathrm{n}-1 .
\end{array}\right.
$$

Now, using the boundary conditions $y^{\prime \prime \prime}(\mathrm{c})=\mathrm{A}_{3}$ and $\mathrm{y}^{\prime \prime \prime}(\mathrm{d})=$ $\mathrm{B}_{3}$ of (2) to the approximate solution $\mathrm{y}(\mathrm{x})$ in (12), we get

$$
\begin{aligned}
\mathrm{y}^{\prime \prime \prime}(\mathrm{c}) & =\mathrm{y}^{\prime \prime \prime}\left(\mathrm{x}_{0}\right)=\mathrm{w}_{3}{ }^{\prime \prime \prime}\left(\mathrm{x}_{0}\right)+\alpha_{1} \mathrm{R}_{1}{ }^{\prime \prime \prime}\left(\mathrm{x}_{0}\right)+\alpha_{2} \mathrm{R}_{2}{ }^{\prime \prime \prime}\left(\mathrm{x}_{0}\right) \\
& =\mathrm{A}_{3} \\
\mathrm{y}^{\prime \prime \prime}(\mathrm{d}) & =\mathrm{y}^{\prime \prime \prime}\left(\mathrm{x}_{\mathrm{n}}\right)=\mathrm{w}_{3}{ }^{\prime \prime \prime}\left(\mathrm{x}_{\mathrm{n}}\right)+\alpha_{\mathrm{n}-2} \mathrm{R}_{\mathrm{n}-2}{ }^{\prime \prime \prime}\left(\mathrm{x}_{\mathrm{n}}\right)+\alpha_{\mathrm{n}-1} \mathrm{R}_{\mathrm{n}-1}{ }^{\prime \prime \prime}\left(\mathrm{x}_{\mathrm{n}}\right) \\
& =\mathrm{B}_{3} .
\end{aligned}
$$

Now, eliminating $\alpha_{1}$ and $\alpha_{n-1}$ from the equations (12), (13) and (14), we get the approximation for $y(x)$ as

$$
\mathrm{y}(\mathrm{x})=\mathrm{w}_{4}(\mathrm{x})+\sum_{j=2}^{n-2} \alpha_{j} \mathrm{~S}_{\mathrm{j}}(\mathrm{x})
$$

where

$$
\begin{aligned}
& \mathrm{w}_{4}(\mathrm{x})=\mathrm{w}_{3}(\mathrm{x})+\frac{\mathrm{A}_{3}-\mathrm{w}_{3}^{\prime \prime \prime}\left(\mathrm{x}_{0}\right)}{\mathrm{R}_{1}{ }^{\prime \prime}\left(\mathrm{x}_{0}\right)} \mathrm{R}_{1}(\mathrm{x}) \\
& +\frac{\mathrm{B}_{3}-\mathrm{w}_{3}^{\prime \prime \prime}\left(\mathrm{x}_{\mathrm{n}}\right)}{\mathrm{R}_{\mathrm{n}-1}^{\prime \prime \prime}\left(\mathrm{x}_{\mathrm{n}}\right)} \mathrm{R}_{\mathrm{n}-1}(\mathrm{x})
\end{aligned}
$$

and

$$
S_{j}(x)=\left\{\begin{array}{l}
\mathrm{R}_{\mathrm{j}}(\mathrm{x})-\frac{\mathrm{R}_{\mathrm{j}}^{\prime \prime \prime}\left(\mathrm{x}_{0}\right)}{\mathrm{R}_{1}^{\prime \prime \prime}\left(\mathrm{x}_{0}\right)} \mathrm{R}_{1}(\mathrm{x}), \text { for } \mathrm{j}=2 \\
\mathrm{R}_{\mathrm{j}}(\mathrm{x}), \quad \text { for } \mathrm{j}=3,4, \ldots, \mathrm{n}-3 \\
\mathrm{R}_{\mathrm{j}}(\mathrm{x})-\frac{\mathrm{R}_{\mathrm{j}}^{\prime \prime \prime}\left(\mathrm{x}_{\mathrm{n}}\right)}{\mathrm{R}_{\mathrm{n}-1}{ }^{\prime \prime \prime}\left(\mathrm{x}_{\mathrm{n}}\right)} \mathrm{R}_{\mathrm{n}-1}(\mathrm{x}), \text { for } \mathrm{j}=\mathrm{n}-2 .
\end{array}\right.
$$

Now, using the boundary conditions $\mathrm{y}^{(4)}(\mathrm{c})=\mathrm{A}_{4}$ and $\mathrm{y}^{(4)}(\mathrm{d})=$ $\mathrm{B}_{4}$ of (2) to the approximate solution $\mathrm{y}(\mathrm{x})$ in (15), we get

$\mathrm{y}^{(4)}(\mathrm{c})=\mathrm{y}^{(4)}\left(\mathrm{x}_{0}\right)=\mathrm{w}_{4}{ }^{(4)}\left(\mathrm{x}_{0}\right)+\alpha_{2} \mathrm{~S}_{2}{ }^{(4)}\left(\mathrm{x}_{0}\right)=\mathrm{A}_{4}$

$\mathrm{y}^{(4)}(\mathrm{d})=\mathrm{y}^{(4)}\left(\mathrm{x}_{\mathrm{n}}\right)=\mathrm{w}_{4}{ }^{(4)}\left(\mathrm{x}_{\mathrm{n}}\right)+\alpha_{\mathrm{n}-2} \mathrm{~S}_{\mathrm{n}-2}{ }^{(4)}\left(\mathrm{x}_{\mathrm{n}}\right)=\mathrm{B}_{4}$.

Now, eliminating $\alpha_{2}$ and $\alpha_{\mathrm{n}-2}$ from the equations (15), (16) and (17), we get the approximation for $\mathrm{y}(\mathrm{x})$ as $\mathrm{y}(\mathrm{x})=\mathrm{w}(\mathrm{x})+\sum_{\mathrm{j}=3}^{n-3} \alpha_{\mathrm{j}} \tilde{\mathrm{B}}_{\mathrm{j}}(\mathrm{x})$

where

$$
\begin{aligned}
& \mathrm{w}(\mathrm{x})=\mathrm{w}_{4}(\mathrm{x})+\frac{\mathrm{A}_{4}-\mathrm{w}_{4}{ }^{(4)}\left(\mathrm{x}_{0}\right)}{\mathrm{S}_{2}{ }^{(4)}\left(\mathrm{x}_{0}\right)} \mathrm{S}_{2}(\mathrm{x}) \\
& +\frac{\mathrm{B}_{4}-\mathrm{w}_{4}{ }^{(4)}\left(\mathrm{x}_{\mathrm{n}}\right)}{\mathrm{S}_{\mathrm{n}-2}{ }^{(4)}\left(\mathrm{x}_{\mathrm{n}}\right)} \mathrm{S}_{\mathrm{n}-2}(\mathrm{x})
\end{aligned}
$$

and

$\widetilde{\mathbf{B}}_{\mathrm{j}}(\mathrm{x})=S_{j}(x), \quad$ for $\mathrm{j}=3,4, \ldots, \mathrm{n}-3$.

Now the new basis functions for the approximation $\mathrm{y}(\mathrm{x})$ are $\left\{\tilde{B}_{j}(x), j=3,4, \ldots, n-3\right\}$ and they are in number match with the number of selected collocated points. Since the approximation for $\mathrm{y}(\mathrm{x})$ in (18) is a quintic approximation, let us approximate $\mathrm{y}^{(5)}, \mathrm{y}^{(6)}, \mathrm{y}^{(7)}, \mathrm{y}^{(8)}, \mathrm{y}^{(9)}$ and $\mathrm{y}^{(10)}$ at the selected collocation points with central differences as

$$
\begin{aligned}
& y_{i}{ }^{(5)}=\left(y_{i+1}{ }^{(4)}-y_{i-1}{ }^{(4)}\right) /(2 h) \\
& y_{i}{ }^{(6)}=\left(y_{i+1}{ }^{(4)}-2 y_{i}{ }^{(4)}+y_{i-1}{ }^{(4)}\right) / h^{2} \\
& y_{i}{ }^{(7)}=\left(y_{i+2}{ }^{(4)}-2 y_{i+1}{ }^{(4)}+2 y_{i-1}{ }^{(4)}-y_{i-2}{ }^{(4)}\right) /\left(2 h^{3}\right)
\end{aligned}
$$


$y_{i}^{(8)}=\left(y_{i+2}^{(4)}-4 y_{i+1}^{(4)}+6 y_{i}^{(4)}-4 y_{i-1}^{(4)}\right.$

$\left.+y_{i-2}^{(4)}\right) / h^{4}$

$y_{i}{ }^{(9)}=\left(y_{i+3}{ }^{(4)}-4 y_{i+2}{ }^{(4)}+5 y_{i+1}{ }^{(4)}-5 y_{i-1}{ }^{(4)}\right.$

$\left.+4 y_{i-2}^{(4)}-y_{i-3}^{(4)}\right) /\left(2 h^{5}\right)$

$y_{i}{ }^{(10)}=\left(y_{i+3}{ }^{(4)}-6 y_{i+2}{ }^{(4)}+15 y_{i+1}{ }^{(4)}-20 y_{i}{ }^{(4)}\right.$

$\left.+15 y_{i-1}{ }^{(4)}-6 y_{i-2}{ }^{(4)}+y_{i-3}{ }^{(4)}\right) / h^{6}$

where

$\mathrm{y}_{i}=y\left(x_{i}\right)=\mathrm{w}\left(\mathrm{x}_{\mathrm{i}}\right)+\sum_{\mathrm{j}=3}^{n-3} \alpha_{\mathrm{j}} \widetilde{\mathrm{B}}_{\mathrm{j}}\left(\mathrm{x}_{\mathrm{i}}\right)$.

Now applying collocation method to (1), we get

$a_{0}\left(x_{i}\right) y_{i}{ }^{(10)}+a_{1}\left(x_{i}\right) y_{i}{ }^{(9)}+a_{2}\left(x_{i}\right) y_{i}{ }^{(8)}+a_{3}\left(x_{i}\right) y_{i}{ }^{(7)}$

$+a_{4}\left(x_{i}\right) y_{i}{ }^{(6)}+a_{5}\left(x_{i}\right) y_{i}{ }^{(5)}+a_{6}\left(x_{i}\right) y_{i}{ }^{(4)}+a_{7}\left(x_{i}\right) y_{i}{ }^{\prime \prime}$

$+a_{8}\left(x_{i}\right) y_{i}{ }^{\prime}+a_{9}\left(x_{i}\right) y_{i}^{\prime}+a_{10}\left(x_{i}\right) y_{i}=b\left(x_{i}\right)$

for $i=3,4, \ldots, n-3$.

Using (19) and (20) in (21) and after rearranging the terms, we get the system of equations which were written in the matrix form as

$$
\mathbf{A} \alpha=\mathbf{B}
$$

where

$$
\mathbf{A}=\left[\mathrm{a}_{\mathrm{ij}}\right]
$$

$a_{i j}=\tilde{B}_{j}{ }^{(4)}\left(x_{i-3}\right)\left(\frac{a_{0}\left(x_{i}\right)}{h^{6}}-\frac{a_{1}\left(x_{i}\right)}{2 h^{5}}\right)$

$+\tilde{B}_{j}{ }^{(4)}\left(x_{i-2}\right)\left(-6 \frac{a_{0}\left(x_{i}\right)}{h^{6}}+4 \frac{a_{1}\left(x_{i}\right)}{2 h^{5}}+\frac{a_{2}\left(x_{i}\right)}{h^{4}}-\frac{a_{3}\left(x_{i}\right)}{2 h^{3}}\right)$

$+\tilde{B}_{j}{ }^{(4)}\left(x_{i-1}\right)\left(15 \frac{a_{0}\left(x_{i}\right)}{h^{6}}-5 \frac{a_{1}\left(x_{i}\right)}{2 h^{5}}-4 \frac{a_{2}\left(x_{i}\right)}{h^{4}}\right.$

$\left.+2 \frac{a_{3}\left(x_{i}\right)}{2 h^{3}}+\frac{a_{4}\left(x_{i}\right)}{h^{2}}-\frac{a_{5}\left(x_{i}\right)}{2 h}\right)$

$+\tilde{B}_{j}{ }^{(4)}\left(x_{i}\right)\left(-20 \frac{a_{0}\left(x_{i}\right)}{h^{6}}+6 \frac{a_{2}\left(x_{i}\right)}{h^{4}}-2 \frac{a_{4}\left(x_{i}\right)}{h^{2}}+a_{6}\left(x_{i}\right)\right)$

$+\tilde{B}_{j}{ }^{(4)}\left(x_{i+1}\right)\left(15 \frac{a_{0}\left(x_{i}\right)}{h^{6}}+5 \frac{a_{1}\left(x_{i}\right)}{2 h^{5}}-4 \frac{a_{2}\left(x_{i}\right)}{h^{4}}\right.$

$\left.-2 \frac{a_{3}\left(x_{i}\right)}{2 h^{3}}+\frac{a_{4}\left(x_{i}\right)}{h^{2}}+\frac{a_{5}\left(x_{i}\right)}{2 h}\right)$

$\tilde{+B}_{j}{ }^{(4)}\left(x_{i+2}\right)\left(-6 \frac{a_{0}\left(x_{i}\right)}{h^{6}}-4 \frac{a_{1}\left(x_{i}\right)}{2 h^{5}}+\frac{a_{2}\left(x_{i}\right)}{h^{4}}+\frac{a_{3}\left(x_{i}\right)}{2 h^{3}}\right)$

$+\tilde{B}_{j}^{(4)}\left(x_{i+3}\right)\left(\frac{a_{0}\left(x_{i}\right)}{h^{6}}+\frac{a_{1}\left(x_{i}\right)}{2 h^{5}}\right)$

$+\tilde{B}_{j}{ }^{\prime \prime}\left(x_{i}\right) a_{7}\left(x_{i}\right)+\tilde{B}_{j}{ }^{\prime \prime}\left(x_{i}\right) a_{8}\left(x_{i}\right)$

$+\tilde{B}_{j}{ }^{\prime}\left(x_{i}\right) a_{9}\left(x_{i}\right)+\tilde{B}_{j}\left(x_{i}\right) a_{10}\left(x_{i}\right)$ for $\mathrm{i}=3,4, \ldots, \mathrm{n}-3, \quad \mathrm{j}=3,4, \ldots, \mathrm{n}-3$.

$\mathrm{B}=\left[\mathrm{b}_{\mathrm{i}}\right]$

$b_{i}=b\left(x_{i}\right)-\left[w^{(4)}\left(x_{i-3}\right)\left(\frac{a_{0}\left(x_{i}\right)}{h^{6}}-\frac{a_{1}\left(x_{i}\right)}{2 h^{5}}\right)\right.$

$+w^{(4)}\left(x_{i-2}\right)\left(-6 \frac{a_{0}\left(x_{i}\right)}{h^{6}}+4 \frac{a_{1}\left(x_{i}\right)}{2 h^{5}}+\frac{a_{2}\left(x_{i}\right)}{h^{4}}-\frac{a_{3}\left(x_{i}\right)}{2 h^{3}}\right)$

$$
\begin{aligned}
& +w^{(4)}\left(x_{i-1}\right)\left(15 \frac{a_{0}\left(x_{i}\right)}{h^{6}}-5 \frac{a_{1}\left(x_{i}\right)}{2 h^{5}}-4 \frac{a_{2}\left(x_{i}\right)}{h^{4}}\right. \\
& \left.+2 \frac{a_{3}\left(x_{i}\right)}{2 h^{3}}+\frac{a_{4}\left(x_{i}\right)}{h^{2}}-\frac{a_{5}\left(x_{i}\right)}{2 h}\right) \\
& +w^{(4)}\left(x_{i}\right)\left(-20 \frac{a_{0}\left(x_{i}\right)}{h^{6}}+6 \frac{a_{2}\left(x_{i}\right)}{h^{4}}-2 \frac{a_{4}\left(x_{i}\right)}{h^{2}}+a_{6}\left(x_{i}\right)\right) \\
& +w^{(4)}\left(x_{i+1}\right)\left(15 \frac{a_{0}\left(x_{i}\right)}{h^{6}}+5 \frac{a_{1}\left(x_{i}\right)}{2 h^{5}}-4 \frac{a_{2}\left(x_{i}\right)}{h^{4}}\right. \\
& \left.-2 \frac{a_{3}\left(x_{i}\right)}{2 h^{3}}+\frac{a_{4}\left(x_{i}\right)}{h^{2}}+\frac{a_{5}\left(x_{i}\right)}{2 h}\right) \\
& +w^{(4)}\left(x_{i+2}\right)\left(-6 \frac{a_{0}\left(x_{i}\right)}{h^{6}}-4 \frac{a_{1}\left(x_{i}\right)}{2 h^{5}}+\frac{a_{2}\left(x_{i}\right)}{h^{4}}+\frac{a_{3}\left(x_{i}\right)}{2 h^{3}}\right) \\
& +w^{(4)}\left(x_{i+3}\right)\left(\frac{a_{0}\left(x_{i}\right)}{h^{6}}+\frac{a_{1}\left(x_{i}\right)}{2 h^{5}}\right) \\
& +w^{\prime \prime \prime}\left(x_{i}\right) a_{7}\left(x_{i}\right)+w^{\prime \prime}\left(x_{i}\right) a_{8}\left(x_{i}\right) \\
& \left.\left.+w^{\prime}\left(x_{i}\right) a_{9}\left(x_{i}\right)+w_{(x}\right) a_{10}\left(x_{i}\right)\right] \\
& \text { for i }=3,4, \ldots, \mathrm{n}-3 \\
& \alpha=\left[\alpha_{3}, \alpha_{4, \ldots,} \alpha_{\mathrm{n}-3}\right]^{\top} .
\end{aligned}
$$

\section{SOLUTION PROCEDURE TO FIND THE NODAL PARAMETERS}

The basis function $\tilde{B}_{i}(x)$ is defined only in the interval $\left[\mathrm{x}_{\mathrm{i}-3,}\right.$, $\mathrm{x}_{\mathrm{i}+3}$ ] and outside of this interval it is zero. Also at the end points of the interval $\left[\mathrm{x}_{\mathrm{i}-3}, \mathrm{x}_{\mathrm{i}+3}\right]$ the basis function $\tilde{B}_{i}(x)$ vanishes. Therefore, $\tilde{B}_{i}(x)$ is having non-vanishing values at the mesh points $\mathrm{x}_{\mathrm{i}-2,}, \mathrm{X}_{\mathrm{i}-1}, \mathrm{X}_{\mathrm{i}}, \mathrm{X}_{\mathrm{i}+1}, \mathrm{X}_{\mathrm{i}+2}$ and zero at the other mesh points. The first four derivatives of $\tilde{B}_{i}(x)$ also have the same nature at the mesh points as in the case of $\tilde{B}_{i}(x)$. Using these facts, we can say that the matrix A defined in (23) is an eleven diagonal band matrix. Therefore, the system of equations (22) is an eleven diagonal band system in $\alpha_{i}^{\prime}$ s. The nodal parameters $\alpha_{\mathrm{i}}^{\prime}$ s can be obtained by using band matrix solution package. We have used the FORTRAN-90 programming to solve the boundary value problem (1)-(2) by the proposed method.

\section{NUMERICAL EXAMPLES}

To demonstrate the applicability of the proposed method for solving the tenth order boundary value problems of type (1)(2), we considered seven examples of which four are linear and three are non linear boundary value problems. Numerical results for each problem are presented in tabular forms and compared with the exact solutions available in the literature.

Example 1 Consider the linear boundary value problem

$\mathrm{y}^{(10)}-\left(\mathrm{x}^{2}-2 \mathrm{x}\right) \mathrm{y}=10 \cos \mathrm{x}-(\mathrm{x}-1)^{3} \sin \mathrm{x}, \quad-1<\mathrm{x}<1$

subject to the boundary conditions
$\mathrm{y}(-1)=2 \sin 1$,
$\mathrm{y}(1)=0$,
$y^{\prime}(-1)=-2 \cos 1-\sin 1, \quad y^{\prime}(1)=\sin 1$,
$\mathrm{y}^{\prime \prime}(-1)=2 \cos 1-2 \sin 1, \quad \mathrm{y}^{\prime \prime}(1)=2 \cos 1$,
$y^{\prime \prime \prime}(-1)=2 \cos 1+3 \sin 1, \quad y^{\prime \prime \prime}(1)=-3 \sin 1$, 
$y^{(4)}(-1)=-4 \cos 1+2 \sin 1, \quad y^{(4)}(1)=-4 \cos 1$.

The exact solution for the above problem is given by

$y(x)=(x-1) \sin x$. The proposed method is tested on this problem where the domain $[-1,1]$ is divided into 11 equal subintervals. Numerical results for this problem are shown in Table 1. The maximum absolute error obtained by the proposed method is $1.047821 \times 10^{-5}$.

Table 1. Numerical results for Example 1

\begin{tabular}{|c|c|c|}
\hline $\mathrm{x}$ & Exact Solution & $\begin{array}{c}\text { Absolute error } \\
\text { by the proposed method }\end{array}$ \\
\hline-0.8 & 1.291241 & $2.622604 \mathrm{E}-06$ \\
\hline-0.6 & $9.034280 \mathrm{E}-01$ & $5.960464 \mathrm{E}-08$ \\
\hline-0.4 & $5.451856 \mathrm{E}-01$ & $4.589558 \mathrm{E}-06$ \\
\hline-0.2 & $2.384032 \mathrm{E}-01$ & $8.985400 \mathrm{E}-06$ \\
\hline 0.0 & 0.0000000000 & $1.047821 \mathrm{E}-05$ \\
\hline 0.2 & $-1.589355 \mathrm{E}-01$ & $7.331371 \mathrm{E}-06$ \\
\hline 0.4 & $-2.336510 \mathrm{E}-01$ & $1.743436 \mathrm{E}-06$ \\
\hline 0.6 & $-2.258570 \mathrm{E}-01$ & $1.341105 \mathrm{E}-06$ \\
\hline 0.8 & $-1.434712 \mathrm{E}-01$ & $1.624227 \mathrm{E}-06$ \\
\hline
\end{tabular}

Example 2 Consider the linear boundary value problem $\mathrm{y}^{(10)}+5 \mathrm{y}=10 \cos \mathrm{x}+4(\mathrm{x}-1) \sin \mathrm{x}, \quad 0<\mathrm{x}<1$

subject to the boundary conditions

$$
\begin{array}{ll}
\mathrm{y}(0)=0, & \mathrm{y}(1)=0, \\
\mathrm{y}^{\prime}(0)=-1, & \mathrm{y}^{\prime}(1)=\sin 1, \\
\mathrm{y}^{\prime \prime}(0)=2, & \mathrm{y}^{\prime \prime}(1)=2 \cos 1, \\
\mathrm{y}^{\prime \prime \prime}(0)=1, & \mathrm{y}^{\prime \prime}(1)=-3 \sin 1, \\
\mathrm{y}^{(4)}(0)=-4, & \mathrm{y}^{(4)}(1)=-4 \cos 1 .
\end{array}
$$

The exact solution for the above problem is given by $\mathrm{y}(\mathrm{x})=(\mathrm{x}-$ $1) \sin \mathrm{x}$. The proposed method is tested on this problem where the domain [0,1] is divided into 11 equal subintervals. Numerical results for this problem are shown in Table 2. The maximum absolute error obtained by the proposed method is $7.942319 \times 10^{-6}$.

Table 2. Numerical results for Example 2

\begin{tabular}{|c|c|c|}
\hline $\mathrm{x}$ & Exact Solution & $\begin{array}{c}\text { Absolute error } \\
\text { by the proposed method }\end{array}$ \\
\hline 0.1 & $-8.985008 \mathrm{E}-02$ & $1.907349 \mathrm{E}-06$ \\
\hline 0.2 & $-1.589355 \mathrm{E}-01$ & $6.705523 \mathrm{E}-07$ \\
\hline 0.3 & $-2.068641 \mathrm{E}-01$ & $3.293157 \mathrm{E}-06$ \\
\hline 0.4 & $-2.336510 \mathrm{E}-01$ & $5.915761 \mathrm{E}-06$ \\
\hline 0.5 & $-2.397128 \mathrm{E}-01$ & $7.450581 \mathrm{E}-06$ \\
\hline 0.6 & $-2.258570 \mathrm{E}-01$ & $7.942319 \mathrm{E}-06$ \\
\hline 0.7 & $-1.932653 \mathrm{E}-01$ & $5.394220 \mathrm{E}-06$ \\
\hline 0.8 & $-1.434712 \mathrm{E}-01$ & $2.756715 \mathrm{E}-06$ \\
\hline 0.9 & $-7.833266 \mathrm{E}-02$ & $1.020730 \mathrm{E}-06$ \\
\hline
\end{tabular}

Example 3 Consider the linear boundary value problem

$$
\mathrm{y}^{(10)}+\mathrm{y}=-10(2 \mathrm{x} \sin \mathrm{x}-9 \cos \mathrm{x}), \quad-1<\mathrm{x}<1
$$

subject to the boundary conditions

$$
\begin{array}{ll}
y(-1)=0, & y(1)=0, \\
y^{\prime}(-1)=-2 \cos 1, & y^{\prime}(1)=2 \cos 1, \\
y^{\prime \prime}(-1)=2 \cos 1-4 \sin 1, & y^{\prime \prime}(1)=2 \cos 1-4 \sin 1,
\end{array}
$$

$y^{\prime \prime \prime}(-1)=6 \cos 1+6 \sin 1, \quad y^{\prime \prime \prime}(1)=-6 \cos 1-6 \sin 1$,

$y^{(4)}(-1)=-12 \cos 1+8 \sin 1, \quad y^{(4)}(1)=-12 \cos 1+8 \sin 1$.

The exact solution for the above problem is given by $\mathrm{y}(\mathrm{x})$ $=\left(\mathrm{x}^{2}-1\right) \cos \mathrm{x}$. The proposed method is tested on this problem where the domain $[-1,1]$ is divided into 11 equal subintervals. Numerical results for this problem are shown in Table 3 . The maximum absolute error obtained by the proposed method is $7.688999 \times 10^{-5}$.

Table 3. Numerical results for Example 3

\begin{tabular}{|c|c|c|}
\hline$x$ & Exact Solution & $\begin{array}{c}\text { Absolute error } \\
\text { by the proposed method }\end{array}$ \\
\hline-0.8 & $-2.508144 \mathrm{E}-01$ & $7.122755 \mathrm{E}-06$ \\
\hline-0.6 & $-5.282148 \mathrm{E}-01$ & $1.859665 \mathrm{E}-05$ \\
\hline-0.4 & $-7.736912 \mathrm{E}-01$ & $4.291534 \mathrm{E}-05$ \\
\hline-0.2 & $-9.408639 \mathrm{E}-01$ & $6.842613 \mathrm{E}-05$ \\
\hline 0.0 & -1.000000 & $7.688999 \mathrm{E}-05$ \\
\hline 0.2 & $-9.408639 \mathrm{E}-01$ & $6.031990 \mathrm{E}-05$ \\
\hline 0.4 & $-7.736912 \mathrm{E}-01$ & $3.105402 \mathrm{E}-05$ \\
\hline 0.6 & $-5.282148 \mathrm{E}-01$ & $8.761883 \mathrm{E}-06$ \\
\hline 0.8 & $-2.508144 \mathrm{E}-01$ & $1.192093 \mathrm{E}-07$ \\
\hline
\end{tabular}

Example 4 Consider the linear boundary value problem

$\mathrm{y}^{(10)}-\mathrm{y}^{\prime \prime}=-8 \mathrm{e}^{\mathrm{x}}$,

$0<\mathrm{x}<1$

subject to the boundary conditions

$$
\begin{array}{ll}
\mathrm{y}(0)=1, & \mathrm{y}(1)=0, \\
\mathrm{y}^{\prime}(0)=0, & \mathrm{y}^{\prime}(1)=-\mathrm{e}, \\
\mathrm{y}^{\prime \prime}(0)=-1, & \mathrm{y}^{\prime \prime}(1)=-2 \mathrm{e}, \\
\mathrm{y}^{\prime \prime \prime}(0)=-2, & \mathrm{y}^{\prime \prime \prime}(1)=-3 \mathrm{e}, \\
\mathrm{y}^{(4)}(0)=-3, & \mathrm{y}^{(4)}(1)=-4 \mathrm{e} .
\end{array}
$$

The exact solution for the above problem is given by $\mathrm{y}(\mathrm{x})=$ $(1-\mathrm{x}) \mathrm{e}^{\mathrm{x}}$. The proposed method is tested on this problem where the domain $[0,1]$ is divided into 11 equal subintervals. Numerical results for this problem are shown in Table 4 . The maximum absolute error obtained by the proposed method is $1.430511 \times 10^{-5}$

Table 4. Numerical results for Example 4

\begin{tabular}{|c|c|c|}
\hline$x$ & Exact Solution & $\begin{array}{c}\text { Absolute error } \\
\text { by the proposed method }\end{array}$ \\
\hline 0.1 & $9.946538 \mathrm{E}-01$ & $8.821487 \mathrm{E}-06$ \\
\hline 0.2 & $9.771222 \mathrm{E}-01$ & $8.642673 \mathrm{E}-06$ \\
\hline 0.3 & $9.449012 \mathrm{E}-01$ & $2.920628 \mathrm{E}-06$ \\
\hline 0.4 & $8.950948 \mathrm{E}-01$ & $5.960464 \mathrm{E}-07$ \\
\hline 0.5 & $8.243606 \mathrm{E}-01$ & $6.735325 \mathrm{E}-06$ \\
\hline 0.6 & $7.288475 \mathrm{E}-01$ & $1.430511 \mathrm{E}-05$ \\
\hline 0.7 & $6.041259 \mathrm{E}-01$ & $1.269579 \mathrm{E}-05$ \\
\hline 0.8 & $4.451082 \mathrm{E}-01$ & $8.136034 \mathrm{E}-06$ \\
\hline 0.9 & $2.459602 \mathrm{E}-01$ & $3.486872 \mathrm{E}-06$ \\
\hline
\end{tabular}

Example 5 Consider the nonlinear boundary value problem

$$
\mathrm{y}^{(10)}=\mathrm{e}^{-\mathrm{x}} \mathrm{y}^{2}(\mathrm{x}), \quad 0<\mathrm{x}<1
$$

subject to the boundary conditions

$$
\begin{array}{ll}
\mathrm{y}(0)=1, & \mathrm{y}(1)=\mathrm{e}, \\
\mathrm{y}^{\prime}(0)=1, & \mathrm{y}^{\prime}(1)=\mathrm{e}, \\
\mathrm{y}^{\prime \prime}(0)=1, & \mathrm{y}^{\prime \prime}(1)=\mathrm{e},
\end{array}
$$


$\mathrm{y}^{\prime \prime \prime}(0)=1$,

$\mathrm{y}^{\prime \prime \prime}(1)=\mathrm{e}$,

$\mathrm{y}^{(4)}(0)=1$,

$y^{(4)}(1)=e$.

The exact solution for the above problem is given by $\mathrm{y}(\mathrm{x})=$ $\mathrm{e}^{\mathrm{x}}$. This nonlinear boundary value problem is converted into a sequence of linear boundary value problems generated by quasilinearization technique [15] as

$\mathrm{y}_{(\mathrm{n}+1)}{ }^{(10)}+\left[-2 \mathrm{e}^{-\mathrm{x}} \mathrm{y}_{(\mathrm{n})}\right] \mathrm{y}_{(\mathrm{n}+1)}=-\mathrm{e}^{-\mathrm{x}} \mathrm{y}_{(\mathrm{n})}{ }^{2}$, for $n=0,1,2 \ldots$

subject to the boundary conditions

$$
\begin{array}{ll}
\mathrm{y}_{(\mathrm{n}+1)}(0)=1, & \mathrm{y}_{(\mathrm{n}+1)}(1)=\mathrm{e}, \\
\left.\mathrm{y}_{(\mathrm{n}+1)}\right)^{\prime}(0)=1, & \left.\mathrm{y}_{(\mathrm{n}+1)}\right)^{\prime}(1)=\mathrm{e}, \\
\left.\mathrm{y}_{(\mathrm{n}+1)^{\prime}}\right)^{\prime \prime}(0)=1, & \mathrm{y}_{(\mathrm{n}+1)}{ }^{\prime}(1)=\mathrm{e}, \\
\left.\mathrm{y}_{(\mathrm{n}+1)}\right)^{\prime \prime(0)}=1, & \left.\mathrm{y}_{(\mathrm{n}+1)}\right)^{\prime \prime}(1)=\mathrm{e}, \\
\mathrm{y}_{(\mathrm{n}+1)}{ }^{(4)}(0)=1, & \mathrm{y}_{(\mathrm{n}+1)}(4) \\
\end{array}
$$

Here $\mathrm{y}_{(\mathrm{n}+1)}$ is the $(\mathrm{n}+1)^{\text {th }}$ approximation for $\mathrm{y}$. The domain [0, $1]$ is divided into 11 equal subintervals and the proposed method is applied to the sequence of problems (35). Numerical results for this problem are presented in Table 5. The maximum absolute error obtained by the proposed method is $6.794930 \times 10^{-5}$.

Table 5. Numerical results for Example 5

\begin{tabular}{|c|c|c|}
\hline $\mathrm{x}$ & Exact Solution & $\begin{array}{c}\text { Absolute error } \\
\text { by the proposed method }\end{array}$ \\
\hline 0.1 & 1.105171 & $1.251698 \mathrm{E}-05$ \\
\hline 0.2 & 1.221403 & $8.702278 \mathrm{E}-06$ \\
\hline 0.3 & 1.349859 & $2.145767 \mathrm{E}-06$ \\
\hline 0.4 & 1.491825 & $1.132488 \mathrm{E}-05$ \\
\hline 0.5 & 1.648721 & $3.969669 \mathrm{E}-05$ \\
\hline 0.6 & 1.822119 & $5.400181 \mathrm{E}-05$ \\
\hline 0.7 & 2.013753 & $6.794930 \mathrm{E}-05$ \\
\hline 0.8 & 2.225541 & $4.887581 \mathrm{E}-05$ \\
\hline 0.9 & 2.459603 & $2.002716 \mathrm{E}-05$ \\
\hline
\end{tabular}

Example 6 Consider the nonlinear boundary value problem

$$
\mathrm{y}^{(10)}-\mathrm{y}^{\prime \prime \prime}=2 \mathrm{e}^{\mathrm{x}} \mathrm{y}^{2}, \quad 0<\mathrm{x}<1
$$

subject to the boundary conditions

$$
\begin{array}{ll}
\mathrm{y}(0)=1, & \mathrm{y}(1)=1 / \mathrm{e}, \\
\mathrm{y}^{\prime}(0)=-1, & \mathrm{y}^{\prime}(1)=-1 / \mathrm{e}, \\
\mathrm{y}^{\prime \prime}(0)=1, & \mathrm{y}^{\prime \prime}(1)=1 / \mathrm{e}, \\
\mathrm{y}^{\prime \prime \prime}(0)=-1, & \mathrm{y}^{\prime \prime}(1)=-1 / \mathrm{e}, \\
\mathrm{y}^{(4)}(0)=1, & \mathrm{y}^{(4)}(1)=1 / \mathrm{e} .
\end{array}
$$

The exact solution for the above problem is given by $\mathrm{y}(\mathrm{x})=\mathrm{e}^{-\mathrm{x}}$. This nonlinear boundary value problem is converted into a sequence of linear boundary value problems generated by quasilinearization technique [15] as

$\mathrm{y}_{(\mathrm{n}+1)}^{(10)}-\mathrm{y}_{(\mathrm{n}+1)}{ }^{\prime \prime}+\left[-4 \mathrm{e}^{\mathrm{x}} \mathrm{y}_{(\mathrm{n})}\right] \mathrm{y}_{(\mathrm{n}+1)}=-2 \mathrm{e}^{\mathrm{x}} \mathrm{y}_{(\mathrm{n})}{ }^{2}$, for $n=0,1,2 \ldots$

subject to the boundary conditions

$$
\begin{array}{ll}
\mathrm{y}_{(\mathrm{n}+1)}(0)=1, & \mathrm{y}_{(\mathrm{n}+1)}(1)=1 / \mathrm{e}, \\
\mathrm{y}_{(\mathrm{n}+1)^{\prime}}(0)=-1, & \mathrm{y}_{(\mathrm{n}+1)^{\prime}}(1)=-1 / \mathrm{e}, \\
\mathrm{y}_{(\mathrm{n}+1)^{\prime}}{ }^{\prime \prime}(0)=1, & \mathrm{y}_{(\mathrm{n}+1)}{ }^{\prime \prime}(1)=1 / \mathrm{e}, \\
\left.\mathrm{y}_{(\mathrm{n}+1)}\right)^{\prime \prime}(0)=-1, & \mathrm{y}_{(\mathrm{n}+1)}{ }^{\prime \prime}(1)=-1 / \mathrm{e}, \\
\mathrm{y}_{(\mathrm{n}+1)}{ }^{(4)}(0)=1, & \mathrm{y}_{(\mathrm{n}+1)}{ }^{(4)}(1)=1 / \mathrm{e}
\end{array}
$$

Here $\mathrm{y}_{(\mathrm{n}+1)}$ is the $(\mathrm{n}+1)^{\text {th }}$ approximation for $\mathrm{y}$. The domain $[0$, 1] is divided into 11 equal subintervals and the proposed method is applied to the sequence of problems (39). Numerical results for this problem are presented in Table 6 . The maximum absolute error obtained by the proposed method is $1.782179 \times 10^{-5}$.

Table 6. Numerical results for Example 6

\begin{tabular}{|c|c|c|}
\hline$x$ & Exact Solution & $\begin{array}{c}\text { Absolute error } \\
\text { by the proposed method }\end{array}$ \\
\hline 0.1 & $9.048374 \mathrm{E}-01$ & $4.827976 \mathrm{E}-06$ \\
\hline 0.2 & $8.187308 \mathrm{E}-01$ & $2.443790 \mathrm{E}-06$ \\
\hline 0.3 & $7.408182 \mathrm{E}-01$ & $1.472235 \mathrm{E}-05$ \\
\hline 0.4 & $6.703200 \mathrm{E}-01$ & $1.782179 \mathrm{E}-05$ \\
\hline 0.5 & $6.065307 \mathrm{E}-01$ & $1.233816 \mathrm{E}-05$ \\
\hline 0.6 & $5.488116 \mathrm{E}-01$ & $4.768372 \mathrm{E}-06$ \\
\hline 0.7 & $4.965853 \mathrm{E}-01$ & $4.589558 \mathrm{E}-06$ \\
\hline 0.8 & $4.493290 \mathrm{E}-01$ & $5.722046 \mathrm{E}-06$ \\
\hline 0.9 & $4.065697 \mathrm{E}-01$ & $2.235174 \mathrm{E}-06$ \\
\hline
\end{tabular}

Example 7 Consider the nonlinear boundary value problem

$$
y^{(10)}=\frac{14175}{4}(\mathrm{x}+\mathrm{y}+1)^{11}, \quad 0<\mathrm{x}<1
$$

subject to the boundary conditions

$$
\begin{array}{ll}
\mathrm{y}(0)=0, & \mathrm{y}(1)=0, \\
\mathrm{y}^{\prime}(0)=-1 / 2, & \mathrm{y}^{\prime}(1)=1, \\
\mathrm{y}^{\prime \prime}(0)=1 / 2, & \mathrm{y}^{\prime \prime}(1)=4, \\
\mathrm{y}^{\prime \prime \prime}(0)=3 / 4, & \mathrm{y}^{\prime \prime \prime}(1)=12, \\
\mathrm{y}^{(4)}(0)=3 / 2, & \mathrm{y}^{(4)}(1)=48 .
\end{array}
$$

The exact solution for the above problem is given by $y(x)=\frac{2}{2-x}-x-1$. This nonlinear boundary value problem is converted into a sequence of linear boundary value problems generated by quasilinearization technique [15] as

$$
\begin{gathered}
y_{(n+1)}^{(10)}+\left[-\frac{14175}{4}(11)\left(x+y_{(n)}+1\right)^{10}\right] y_{(n+1)} \\
=\frac{14175}{4}\left(x+y_{(n)}+1\right)^{10}\left[x+1-10 y_{(n)}\right], \\
\text { for } n=0,1,2 \ldots
\end{gathered}
$$

subject to the boundary conditions

$$
\begin{array}{ll}
\mathrm{y}_{(\mathrm{n}+1)}(0)=0, & \mathrm{y}_{(\mathrm{n}+1)}(1)=0, \\
\mathrm{y}_{(\mathrm{n}+1)}{ }^{\prime}(0)=-1 / 2, & \left.\mathrm{y}_{(\mathrm{n}+1)}\right)^{\prime}(1)=1, \\
\mathrm{y}_{(\mathrm{n}+1)}{ }^{\prime}(0)=1 / 2, & \left.\mathrm{y}_{(\mathrm{n}+1)}\right)^{\prime}(1)=4, \\
\left.\mathrm{y}_{(\mathrm{n}+1)}\right)^{\prime \prime}(0)=3 / 4, & \mathrm{y}_{(\mathrm{n}+1)}{ }^{\prime \prime}(1)=12, \\
\mathrm{y}_{(\mathrm{n}+1)}(4)(0)=3 / 2, & \mathrm{y}_{(\mathrm{n}+1)}(1)
\end{array}
$$

Here $\mathrm{y}_{(\mathrm{n}+1)}$ is the $(\mathrm{n}+1)^{\text {th }}$ approximation for $\mathrm{y}$. The domain [0, 1] is divided into 11 equal subintervals and the proposed method is applied to the sequence of problems (43). Numerical results for this problem are presented in Table 7 . The maximum absolute error obtained by the proposed method is $6.940961 \times 10^{-5}$. 
Table 7. Numerical results for Example 7

\begin{tabular}{|c|c|c|}
\hline $\mathrm{x}$ & Exact Solution & $\begin{array}{c}\text { Absolute error } \\
\text { by the proposed method }\end{array}$ \\
\hline 0.1 & $-4.736842 \mathrm{E}-02$ & $1.322478 \mathrm{E}-06$ \\
\hline 0.2 & $-8.888889 \mathrm{E}-02$ & $4.231930 \mathrm{E}-06$ \\
\hline 0.3 & $-1.235294 \mathrm{E}-01$ & $1.676381 \mathrm{E}-05$ \\
\hline 0.4 & $-1.500000 \mathrm{E}-01$ & $4.245341 \mathrm{E}-05$ \\
\hline 0.5 & $-1.666667 \mathrm{E}-01$ & $6.663799 \mathrm{E}-05$ \\
\hline 0.6 & $-1.714286 \mathrm{E}-01$ & $6.940961 \mathrm{E}-05$ \\
\hline 0.7 & $-1.615385 \mathrm{E}-01$ & $4.750490 \mathrm{E}-05$ \\
\hline 0.8 & $-1.333333 \mathrm{E}-01$ & $1.643598 \mathrm{E}-05$ \\
\hline 0.9 & $-8.181816 \mathrm{E}-02$ & $2.607703 \mathrm{E}-07$ \\
\hline
\end{tabular}

\section{CONCLUSIONS}

In this paper, we have developed a collocation method with quintic B-splines as basis functions to solve tenth order boundary value problems. Here we have taken internal mesh points $\mathrm{x}_{3}, \mathrm{x}_{4}, \ldots, \mathrm{x}_{\mathrm{n}-3}$ as the selected collocation points. The quintic B-spline basis set has been redefined into a new set of basis functions which in number match with the number of selected collocation points. The proposed method is applied to solve several number of linear and non-linear problems to test the efficiency of the method. The numerical results obtained by the proposed method are in good agreement with the exact solutions available in the literature. The objective of this paper is to present a simple method to solve a tenth order boundary value problem and its easiness for implementation.

\section{REFERENCES}

[1] Chandrasekhar. S, 1961, Hydro dynamic and Hydro magnetic Stability, Clarendon Press, Oxford, (Reprinted: Dover Books, New York. 1981) .

[2] Agarwal. R.P, 1986, Boundary Value Problems for higherorder differential equations, World Scientific, Singapore.

[3] Twizell. E.H, Boutayeb. A, Djidjeli. K. "Numerical methods for eighth, tenth and twelfth-order eigenvalue problems arising in thermal instability". Adv. Comput. Math. 2(1994): 407-436.

[4] Siddiqi. S.S, Twizell. E.H. "Spline solutions of linear tenth-order boundary value problems". International Journal of Computer Mathematics 68(1998): 345-362.

[5] Siddiqi. S.S, Akram. G. "Solution of tenth-order boundary value problems using nonpolynomial spline technique". International Journal of Applied Mathematics and Computation 190(2007): 641-651.

[6] Siddiqi. S.S, Akram. G. "Solution of tenth-order boundary value problems using eleventh degree spline".
International Journal of Applied Mathematics and Computation 165(2007): 115-127.

[7] Erturk. V.S, Momani. S. "A reliable algorithm for solving tenth-order boundary value problems". Num. Algo. 44(2007): 147-158

[8] Siddiqi. S.S, Ikram. G, Zaheer. S. "Solution of Tenth Order Boundary Value Problems using Variational Iteration Technique". J. European Scientific Research 30(2009): 326-347.

[9] Barari. A, Omidvar. M, Najaf. T, Abdoul Ghotbi. T. "Homotopy Perturbation Method for solving tenth order boundary value problems". International Journal of Mathematics and Computation 3(2009): J09.

[10] Scott. M.R, Watts. H.A. "Computional solution of linear two points bvp via orthonormalization". SIMA J. Numer. Anel. 165(1977): 40-70.

[11] Scott. M.R, Watts. H.A, 1976, A systematized collection of codes for solving two-point bvps, Numerical methods for differential systems, Academic Press.

[12] Scott Layne Watson. M.R. "Solving spline-collocation approximations to nonlinear two point boundary value problems by a homotopy method". Appl. Math. Comput. 24(1987): 333-357.

[13] Wazwaz. A.M. "The Modified Adomian Decomposition Method for solving linear and nonlinear boundary value problems of tenth-order and twelfth-order". International Journal of Nonlinear Sciences and Numerical Simulation 1(2000): $17-24$

[14] Djidjeli. K, Twizell. E.H, Boutayeb. A. "Numerical methods for special nonlinear boundary-value problems of order 2m". Journal of Computational and Applied Mathematics 47(1993): 35-45.

[15] Bellman. R.E, Kalaba. R.E, 1965, Qusilinearization and Nonlinear Boundary value problems, American Elsevier, New York.

[16] Reddy. J.N, 2005, An introduction to the Finite Element Method, $3^{\text {rd }}$ Edition, Tata Mc-GrawHill Publishing Company ltd., New Delhi.

[17] Prenter. P.M, 1989, Splines and Variational Methods, John-Wiley and Sons, New York.

[18] Carl de Boor, 1978, A Practical Guide to Splines, Springer-Verlag.

[19] Schoenberg. I.J, 1966, On Spline Functions, MRC Report 625, University of Wisconsin. 